1.16 to 3.70). Persistent offenders, defined by report of both juvenile and adult arrest, had elevated odds of STI compared to those who were never arrested (adjusted OR: 1.72, 95\% CI 1.06 to 2.80), while those who reported history of arrest either as a juvenile or as an adult but not during both time periods did not have elevated STI risk

Conclusions Adolescents who have very high repeat contact with the criminal justice system, who are convicted as juveniles, or who remain offenders into adulthood are priority populations for STI treatment and prevention. Though the potential for residual confounding especially due to unmeasured mood and personality characteristics is a limitation, the independent associations between juvenile CJI indicators and adult STI suggest that for some, the disruptive effect of juvenile CJI may contribute to a trajectory that results in adulthood STI see Abstract P2-S1.15 Table 1.

\section{P2-S1.16 SOCIAL INFLUENCES ON RELATIONSHIP FIDELITY AND CONCURRENCY PATTERNS AMONG LATINO ADOLESCENTS IN SAN FRANCISCO}

doi:10.1136/sextrans-2011-050108.294

A Minnis, E van Dommelen-Gonzalez. RTI International, San Francisco, USA

Background Our previous research in San Francisco's Mission District, a predominantly Latino neighbourhood characterised by high immigration, poverty, and gang-related violence, highlighted diverse partnership patterns among youth and the role of gang affiliation in high-risk sexual activity and pregnancy. 38\% of Latino youth reported concurrent partnerships.

Methods We conducted 33 semi-structured in-depth interviews with immigrant and US-born male and female Latino youth aged 16-21 to explore how the social environment affected partnership formation and STI risk. Participants were recruited from community venues; interviews were conducted in Spanish or English. Interview transcripts were coded in Atlast.ti. We analysed the following themes: relationship fidelity; STI prevention; and concurrency norms (agreement with the statement, Many Mission youth in a relationship are also seeing someone on the side).

Results Participants were $48 \%$ female; had a mean age of 17.5 years; and $55 \%$ were immigrants from Mexico or Central America. 27 of 30 believed that concurrency is widespread, described as "cheating" (having a "main girl with "friends with benefits' on the side") or resulting from initiating new relationships before ending existing ones or between serial break-ups with a main partner. Infidelity was motivated by machismo, a cultural notion of masculinity that was heightened for men respected for having multiple partners; boredom with a single partner; an ideal that fidelity was only relevant within marriage with cheating expected prior to that; and revenge for known, suspected or pre-emptive cheating. Street gangs introduced distinct reasons for young males preferring multiple, casual partnerships: limited time for relationships because of obligations to the gang first and foremost; disinterest in committed relations to avoid emotional vulnerability given emotional demands of street life; and multiple partners bringing status: "you got more game; you got more reputation." Young women recognised that having partners in a gang brought social prestige and articulated strategies for increasing intimacy (eg, pregnancy) to draw partners away from the street and strengthen commitment to the relationship. Despite a perception of pervasive concurrency, STI risk perception was low.
Conclusions Many relationship norms and expectations held by youth supported concurrency, highlighting the importance of addressing social influences like gangs in STI prevention.

\section{P2-S1.17 EVAluating Native STAND: A PEER EDUCATION CURRICULUM FOR HEALTHY DECISION-MAKING FOR NATIVE YOUTH}

doi:10.1136/sextrans-2011-050108.295

${ }^{1} \mathrm{~L}$ de Ravello, ${ }^{2} \mathrm{~S} \mathrm{C}$ Rushing, ${ }^{1} \mathrm{~S}$ Doshi, ${ }^{3} \mathrm{M}$ U Smith, ${ }^{1} \mathrm{~S}$ Tulloch. ${ }^{1}$ Northwest Portland Area Indian Health Board, Portland, USA; ${ }^{2}$ Centers for Disease Control \& Prevention, Atlanta, USA; ${ }^{3}$ Mercer University, Macon, USA

Background American Indian/Alaska Native (AI/AN) youth are disproportionately affected by a myriad of health and social conditions. Compared to other US teens, AI/AN youth have higher rates of sexually transmitted infections, teen pregnancies, tobacco use, alcohol use, and substance use. Native STAND (Native Students Together Against Negative Decisions), a 29-session peer educator curriculum was developed to address a range of sexual and reproductive health topics, including important communication and peer education skills. It is the first peer educator curriculum for healthy decision making developed for AI/AN youth.

Methods Eighty students attending four Bureau of Indian Education (BIE) boarding schools were selected to be trained as peer educators. The curriculum was delivered by trained adult staff at each school and the sessions occurred over the course of the school year with each session approximately $90 \mathrm{~min}$ in length. A pre- and post-test using Computer Assisted Self-Interviewing (CASI) was administered to the participants. At the end of the program, focus groups with peer educators and school staff and key informant interviews with facilitators and school administration were conducted to identify programmatic strengths and weaknesses and inform final program revisions.

Results Overall, analysis of pre- and post-test CASI data showed students at all four schools experienced increases in talking to peers about sexual health; in STI/HIV prevention and reproductive health knowledge; in the intention to use condoms to avoid pregnancy and STIs; and in condom self-efficacy indices. Focus groups and key informant interviews revealed that facilitators and students felt they learnt a lot through the curriculum and enjoyed the program; they saw the program as a badly needed source of clear and honest information; and they voiced their desire to have the program continue.

Conclusions Native STAND was well-received at all four sites by students, facilitators, and school administrators. Almost everyone felt that no topics should be eliminated and that all the curriculum's activities were relevant and important to include to address the unique needs of Native youth. We will incorporate findings from the evaluation into the curriculum and make it widely available.

\section{P2-S1.18 ACCURACY OF SELF-REPORTED CONDOM USE ASSESSED BY THE SEMEN Y-CHROMOSOME BIOMARKER FOR UNPROTECTED SEX}

doi:10.1136/sextrans-2011-050108.296

\begin{abstract}
${ }^{1} \mathrm{~J}$ Rosenbaum, ${ }^{2} \mathrm{~J}$ Melendez, ${ }^{3} \mathrm{E}$ Rose, ${ }^{3} \mathrm{G}$ Wingood, ${ }^{2} \mathrm{~J}$ Zenilman, ${ }^{3} \mathrm{R}$ DiClemente. ${ }^{1}$ University of Maryland School of Public Health, Washington, USA; ${ }^{2}$ Johns Hopkins Medical Institutions, USA; ${ }^{3}$ Emory School of Public Health, USA
\end{abstract}

Background Adolescents may use condoms inconsistently or incorrectly, and may over-report their condom use. Biomarkers offer a 
potential solution to over-report, allowing interventions to target populations at risk from inconsistent condom use. This study uses a biomarker for unprotected sex to evaluate the accuracy of adolescent womens reports of condom use.

Methods The sample comprised 715 African-American female participants in an HIV prevention trial who were ages 15-21, sexually active, and recruited from three urban Atlanta family planning and STI clinics in 2002-2004. At baseline, 6, and 12 months, participants completed a 40 min ACASI interview and were tested for semen Y-chromosome with PCR from a selfadministered vaginal swab. Data analysis used non-parametric tests for trend and clustered logistic regression to predict self-reported pregnancy.

Results At the three waves respectively 30\%, 20\% and 15\% of participants who reported $100 \%$ condom use tested positive for semen Y chromosome. Suspected over-reporters of condom use were more likely to report being pregnant at the following wave than any other category of condom and birth control users: at wave $2,20.4 \%$ of wave 1 suspected over- reporters were pregnant vs $14.2 \%$ of wave 1 condom never-users and $9.8 \%$ of those reporting no birth control at last sex; at wave $3,16.2 \%$ of wave 2 suspected over-reporters were pregnant vs $11.8 \%$ of condom never-users and $10.8 \%$ reporting no birth control at last sex. Suspected over-reporters of unprotected sex were more likely to report pregnancy at the following wave, controlling for frequency of sex, condom use, and oral contraception (OR 3.95 (1.63 to 9.58), 2.64 (0.92 to 7.58)) see Abstract P2-S1.18 Table 1.

Conclusions Respondents who report 100\% condom use but test positive for semen Y-chromosome are at higher risk than other women. These women may be over-reporting their condom use and under-reporting their frequency of sex.

Abstract P2-S1.18 Table 1 Prediction of pregnancy with logistic regression. Analysis was limited to those who participated in all 3 waves of the survey $(n=560)$. Did not report sex past 60 days are those who participated in the wave but did not answer the question how many times they had sex in the past 60 days. Condom use in the past 60 days was imputed from condom use in the past 14 days for these individuals. Predictors of pregnancy are measured in the wave previous to pregnancy

\begin{tabular}{lll}
\hline Variable & OR (95\% Cl) & p \\
\hline Pregnancy at wave 2 & & \\
Suspected over-report & $3.95(1.63$ to 9.58$)$ & 0.002 \\
Times sex past 60 days & $1.02(1.00$ to 1.05$)$ & 0.02 \\
Proportion condom use past 60 days & $0.50(0.21$ to 1.18$)$ & 0.11 \\
Birth control pill & $0.42(0.12$ to 1.40$)$ & 0.16 \\
Did not report sex past 60 days & $0.57(0.15$ to 2.17$)$ & 0.41 \\
Pregnancy at wave 3 & & \\
Suspected over-report & $2.64(0.92$ to 7.58$)$ & 0.07 \\
Times sex past 60 days & $1.04(1.02$ to 1.06$)$ & 0.000 \\
Proportion condom use past 60 days & $0.56(0.24$ to 1.31$)$ & 0.18 \\
Birth control pill & $0.14(0.02$ to 1.08$)$ & 0.06 \\
Did not report sex past 60 days & $1.52(0.57$ to 4.01$)$ & 0.40 \\
\hline
\end{tabular}

\section{Social and behavioural aspects of prevention poster session 2: Female sex workers

P2-S2.01 SUPPORT GROUPS AS A STRATEGY FOR REDUCING HIV
VULNERABILITY AMONG FEMALE SEX WORKERS IN
BELLARY, NORTHERN KARNATAKA, INDIA

doi:10.1136/sextrans-2011-050108.297

${ }^{1} \mathrm{D}$ R Chintada, ${ }^{2} \mathrm{P}$ Pillai, ${ }^{1} \mathrm{~S}$ Mahadevapappa, ${ }^{1} \mathrm{P}$ Mohammed, 'S Isac, ${ }^{1} \mathrm{~B}$ M Ramesh, ${ }^{3} \mathrm{~V}$ Ramappa, ${ }^{1} \mathrm{P}$ Bhattacharjee, ${ }^{4} \mathrm{~S}$ Moses. ${ }^{1}$ Karnataka Health Promotion Trust, Bellary,
India; ${ }^{2}$ Independent consultant, Bangalore, India; ${ }^{3}$ MYRADA, Bellary, India; ${ }^{4}$ University of Manitoba, Winnipeg, Canada

Background The Karnataka Health Promotion Trust implements HIV preventive interventions for female sex workers (FSWs) in Karnataka, South India, in partnership with non-governmental and community-based organizations (NGOs and CBOs). These interventions are supported by the Bill \& Melinda Gates Foundation. Community mobilisation and collectivisation are key vulnerability reduction strategies. In Bellary district, FSWs are encouraged to form site-level groups of 10-12 members which meet regularly. They discuss STIs, HIV, condom negotiation skills, and other important issues. They also support members during crises, and engage in savings and credit activities. We attempted to understand the impact in Bellary of collective membership and the availability of economic support on two measures of safer sex behaviour: clinic attendance and condom use with last client.

Methods Multivariate logistic regression was used to measure the association between the outcome variables and collective membership, using data from a round of integrated behavioural and biological assessments conducted in Bellary in 2008. We also conducted focus group discussions (FGDs) with members and nonmembers of collectives, to understand the reasons for observed associations.

Results $34 \%$ of FSWs were members of collectives. Members were 7 times more likely to have used condoms with their last client (adjusted OR [AOR]: 7.5, 95\% CI 1.9 to 30.1, p<0.05). Additionally, collective members were 4 times more likely to visit an STI clinic than non-members (AOR: 4.6, 95\% CI 2.0 to 10.6, p<0.05). FGDs revealed that alternate sources of economic activity, such as savings enhancement and low interest loans to collective members, reduced sex workers' dependence on exploitative sources of credit, enabling them to better negotiate condom use with clients who resisted unprotected sex. Further, the sex worker collectives helped FSWs to overcome their fears of stigmatisation, creating a more enabling environment that positively influenced access to clinics for STI care. Conclusions Membership in site-level groups considerably increased access of FSWs to program services. Activities to address the economic vulnerability of female sex workers were key factors in enabling greater condom use. Savings and access to credit helped FSWs to reduce their dependency on sex work, and enabled them to more readily negotiate condom use with clients.

\section{P2-S2.02 ROLE OF DECISION MAKERS IN THE LIVES OF MIGRANT SEX WORKERS-FOCUS ON FAMILIES: AN QUALITATIVE ASSESSMENT}

doi:10.1136/sextrans-2011-050108.298

\author{
${ }^{1} \mathrm{~S}$ Ramanaik, ${ }^{1} \mathrm{~V}$ Sabnis, ${ }^{1} \mathrm{~K}$ Gurav, ${ }^{1} \mathrm{P}$ Banandur, ${ }^{1} \mathrm{R}$ Buzdugan, ${ }^{2} \mathrm{~S}$ Halli, ${ }^{2} \mathrm{~J}$ Blanchard \\ ${ }^{1}$ Karnataka Health Promotion Trust, Bangalore, India; ${ }^{2}$ Centre for Global Public Health, \\ University of Manitoba, Winnipeg, Canada
}

Background This study is aimed to understand the role of family members in decision-making process of female sex worker (FSW) migration and its impact on HIV risk, vulnerability and transmission dynamics in Bagalkot, Bijapur and Belgaum districts of Northern Karnataka and southern Maharashtra.

Methods Qualitative in-depth interviews were conducted with family members of the migrant and non-migrant female sex workers (FSWs) as a part of a large cohort study to understand the patterns and determinants of FSW migration from northern Karnataka to southern Maharashtra, South India. The present paper specifically focuses on the findings generated from in-depth, qualitative interviews with 30 family members of migrant and non-migrant (15 each) FSWs who entered into sex work through the traditional" devadasi system. 